\title{
52 \\ Simulation of embedded decision processes in manufacturing systems
}

\author{
S. Cem Karacal \\ Southern Illinois University \\ Mechanical and Industrial Engineering, Edwardsville, IL \\ 62026 \\ skaraca@siue.edu
}

\begin{abstract}
This paper addresses a new unified framework for simulation that includes both physical and decision making processes of manufacturing systems. The developed methodology uses a state-space representation based simulation modeling formalism along with object oriented software design. The model developer perceives the manufacturing system in terms of several physical entities, augmented by a number of decision making elements, each capable of making rational decisions that affect system behavior and contribute to the collective state of the system. The modularity of the user's conceptual model in terms of physical and decision making entities with their programmed and non-programmed decisions and the capability of modeling these entities and processes at different hierarchical levels can vastly improve simulation's role as a decision making tool.
\end{abstract}

Keywords

Manufacturing system simulation, knowledge based simulation, object oriented modeling

Computer simulation modeling is the only viable approach to the detailed analysis and design of complex manufacturing systems. Yet, current simulation languages permit only gross approximations to the actual systems being studied. The simulation methodology still suffers from its traditional deficiencies. During the model development process various system assumptions are hardwired into the 
model code and there are no provisions to simulate the decision processes which are the actual driving forces of purposeful systems. The modification of modeling assumptions usually requires major model overhauls with extensive programming efforts, resulting in minimal ease of model development and code reusability.

The performance of a manufacturing system is highly influenced by control policies used in its operation. The decisions are based on available information, which is often incomplete, inaccurate, and delayed. A decision maker at each level uses heuristics, personal expertise, company rules and policies to arrive at a control decision. Traditional simulation tools do not provide convenient structures for specifying these interactions

This research addresses to the design and implementation of a fundamentally new and superior approach to the modeling of complex systems, based upon a new formalism, state-space representation, and the object oriented programming approach. Specifically, this new simulation modeling environment will permit the distinct and separate specification of physical elements, decision elements, and data/information elements of a manufacturing system. The developed system uses artificial intelligence techniques to imitate the non-programmed decision processes. Realistic models can be constructed at any level of detail desired, and can be retrieved and reused as needed. The ability to perform detailed analysis using this new simulation environment will dramatically reduce the time needed for planning/designing and analyzing complex manufacturing systems. The developed prototype software environment is also compared against conventional simulation approaches using the analytic hierarchy process technique to reach a scale of preference between the two.

The Discrete Event Simulation of Manufacturing Systems (DEMS) formalism developed (Karacal and Mize, 1996a) is a set of conventions for the construction of simulation models. It gives a definite form to how and what can be expressed about a system to be modeled by using set theory and state-space representation for the abstraction. In the end, it generates a science base and a uniform convention of notation and communication. The DEMS formalism is based on five distinct levels for a system entity to be in and can be summarized as follow:

Level 0: At the bottom of the hierarchy lies the Source System represented with:

- Physical components set which contains

Set of physical inputs, $X$

Set of physical outputs, $Y$

Communication channels, $C$

Material ports, $M$

Set of manufacturing operations, $O$

- Observed facts set, $F$

- Set of real numbers for time, $T$ 
- Function read, $\rho$, that maps elements of physical inputs, outputs, or communication channels to the facts set $F$.

Level 1: Next level in the hierarchy is the Data System and expressed with:

- Source System from level 0

- Universe of discourse set, $U$, that represents the meanings for observed facts

- Data set $D$ where each element is defined in terms of a fact from set $F$ and a meaning from $U$

- Function assign, $\theta$, that maps an element of observed facts set and an element of universe of discourse set to data set $D$

- Function time that maps a real number for time to an element of data set to represent temporal data

Level 2: When the relationships and the operations between the elements of the data set are provided, the data system becomes an Information System that is defined through:

- Data System from level 1

- Relation definitions set, R

- Information (internal state) set, I

- Information-generation function $\gamma_{\mathrm{I}}$ that maps elements of data set $\mathrm{D}$ and elements of operations set $\mathrm{O}$ and/or

Relations set $\mathrm{R}$ to information set I

- look function $\lambda$ that is used to obtain information about the states of other system entities

- action function $\alpha$ for programmed decisions based on present internal and external states

- update-internal function $v_{\text {int }}$ to reflect the effects of actions selected on internal state

- update-external function $v_{\text {ext }}$ to reflect the effects of actions selected through $\alpha$ on external state

The dynamic behavior, moving in time from one state to another, of the information system during the simulation is handled by a set of pseudo functions. These functions first observe the internal and external states through functions $\gamma_{\mathrm{I}}$ and $\lambda$ at time $t_{i-1}$. Next, they obtain a programmed decision through action function $\alpha$ at time $t_{i-1}$. Then, the internal and external states are updated using $v_{\text {int }}$ and $v_{\text {ext }}$ functions for next event time $t_{\mathrm{j}}$. This process is repeated as next events take place in simulation.

Level 3: When the entity is transformed from a procedural format to a symbolic format, it is referred to as a Knowledge System and represented with:

- Information System from level 2

- Structured universe of discourse set, Us, that defines the relationships among the meanings 
- knowledge-generation function $\gamma_{k}$ that generates knowledge from a given information set I and structured universe

of discourse set $U_{s}$

- knowledge base set $K$

Level 4: A knowledge system becomes an Intelligent System when a reasoning mechanism is associated with the knowledge base. At this level the entity is abstracted through:

- Knowledge System from level 3

- External knowledge base $B$ that represents the knowledge about the total system

- Goals set $G$ that define the set of goals (production orders) defined for the entity

- Decision set $H$ that represents the set of decisions reached by searching through the knowledge base $K$

- perceive function $\pi$ to asses the knowledge about the total system

- decision function $\delta$ to determine the actions that need to be taken to satisfy the goals defined in set $G$

- update-external-knowledge function $\omega_{\text {ext }}$ to reflect the changes that occurred in the total system as a result of

implementing the decision obtained through decision function $\delta$

- update-internal-knowledge function $\omega_{\text {int }}$ to reflect the changes that occurred in entities internal knowledge base $K$

as a result of implementing the decision obtained through decision function $\delta$.

The dynamic behavior of the intelligent system during the simulation is handled by a set of pseudo functions similar to those defined for the information system. These functions first observe the internal and external knowledge states through functions $\gamma_{\mathbf{k}}$ and $\pi$ at time $t_{i-1}$. Next, they obtain a non-programmed decision through decision function $\delta$ at time $t_{i-1}$ by searching their knowledge bases. Then, the internal and external knowledge bases are updated using $\omega_{\text {int }}$ and $\omega_{\text {ext }}$ functions for time $t_{i}$. Although the dynamic behavior for information and intelligent system levels are similar on the surface, they are very different. The information system level uses information as opposed to knowledge used in intelligent system level. Furthermore, the representation schemes used (procedural format in information, symbolic format in intelligent) in two levels are very different.

\subsection{System Entities}

Two types of entities, decision making entities (DME) and information driven physical entities (IDPE) are defined based on the intelligent and information system levels of the formalism, respectively. The other three levels are mainly definition tools to assess the sets necessary in the operations of DMEs and IDPEs. The DMEs are the basic building blocks of the planning and control system. The IDPEs form the foundation of the manufacturing system. Their 
activities correspond to real physical activities that are taking place on the floor and are direct results of decisions made by DMEs. The interactions between DMEs and IDPEs as well as interactions among DMEs can be established through the use of control, communication and material flow relations. When individual entities are bound together with data, information and knowledge, structural and behavioral representation at the total system level can be easily achieved. Three different classes are defined for the DMEs of a manufacturing system.

i) Entities that deal with the planning and control activities at the system level. These entities utilize the current

state of the system in a filtered form, the work load, and the requirements for product orders.

ii) Entities that plan and control the major activities that need to be carried out and synchronized in order to conform to the plans prepared by the entities of (i ). The type of knowledge utilized by these entities are the current collective state of each unit (shop/department) planned and controlled by each entity. These entities create their own individual plans and release job orders aimed at the satisfaction of product orders coming from above.

iii) Entities that perform planning and control at work-center or cell level. At this level, the orders are further broken down into task orders that need to be synchronized to conform the job orders received from above. This is the lowest level in which intelligent behavior takes place in the hierarchy. The task orders are further broken down into activity orders that are translated into a finite set of ordered physical activities. This is where the logical system connects to the physical system.

A major advantage of these three levels defined for DMEs is its ability to allow the natural analysis of information components. The information and knowledge elements are the main driving and binding forces of the physical and logical systems. Each intelligent entity monitors the orders released by its superior entity, performs its own decision making and planning processes, and releases proper orders to its own subordinates. Thus, each production planning and control problem is solved in its own local domain by the most knowledgeable entity about that area.

\section{3}

OBJECT ORIENTED SIMULATION TOOL

The Smalltalk object oriented programming language (OOP) is chosen for the implementation of the developed formalism and methodology. A hierarchy of object classes that represent system, department, work-center, machine physical entities along with a hierarchy of control and communication objects are defined (Karacal and Mize, 1997a). The following structure summarizes the hierarchy of 
defined Smalltalk object classes. The indentation represents the class subclass relationships.

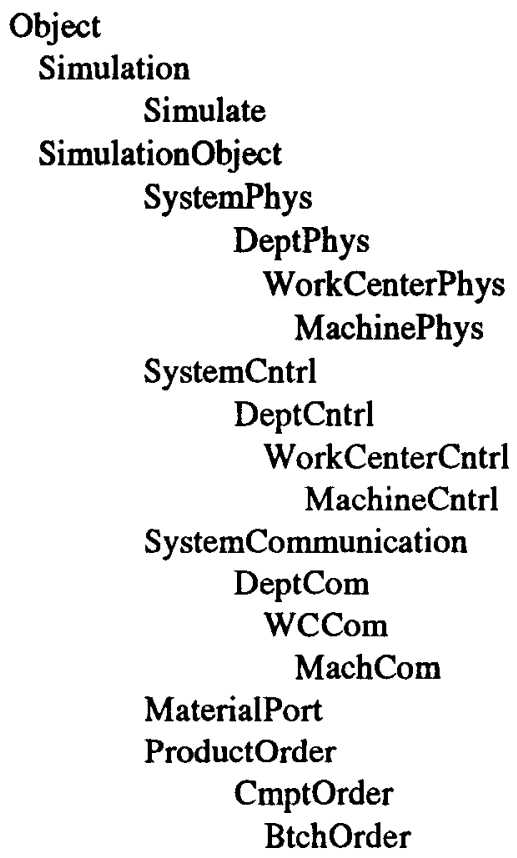

Most of these class names are self explanatory and the instances of them are the basic building blocks of a simulation model. As the model builder defines the physical system configuration, the instances of above classes are automatically created and configured during model building process.

\subsection{Software implementation}

To take full advantage of the inheritance property of OOP, subclasses inheriting their basic behavior from super classes, the whole environment is designed around three abstract tree structures that are transparent to the user during simulation. The first tree, SystemStructure, represents the hierarchical configuration of the physical entities of the manufacturing system being modeled. These entities are defined through instances of physical objects of the above hierarchy and include machine tools, handling devices, etc. and correspond to SourceSystem of the formalism. The software automatically creates a default control entity, communication channel, and material port for each physical entity created and allows the model developer to customize them depending on the capabilities and functions of the physical entity created.

The second tree, ProductDataBase, maintains information on all the products 
produced and their product structure trees including all the relevant information on parts and components. In order to keep track of the dynamic information involved in the simulation, a highly dynamic third tree structure, OrderDataBase, is designed and frequently manipulated during the simulation. This tree holds all the information on product, component, and part orders involved during the simulation period. At the beginning of the simulation, this is a tree consisting of nothing but a root linked to a system control object. As the orders arrive, the branches and sub-branches representing customers, their individual orders, and a break down of those orders are created. The status of orders are updated as the operations and assemblies are performed. When the final operation of a product order is completed, the branch originating from that node is pruned and the related statistics are updated. This process prevents infinite growth of the OrderDataBase.

\section{PRODUCTION CONTROL KNOWLEDGE BASES}

After defining the physical configuration of the system, the next step is the definition of the knowledge bases for each control entity defined in the hierarchy. Three generic knowledge base objects and their inference rules are defined for system, department/shop, and work-center levels and are called systemPlanning, shopPlanning, and workCenterPlanning, respectively. The simulation model developer can express the operational policies and expertise for each intelligent entity during the knowledge base and inference rule definition process. An example class of knowledge bases are defined to implement the production planning and resource allocation policies of a typical make-to-order manufacturing facility. The instances of these knowledge bases are created and linked to the control objects of each intelligent entity in the hierarchy. The inference rules include a certainty value ( 1.0 complete certainty, 0.0 complete uncertainty) that represents how strongly system believes that the decision, the value of parameter action, is the correct one. This value is later used by the system to search for the most certain decision for a given goal. The system utilizes a grammar, based on concepts from formal language theory, to translate customer orders into component orders and then component orders to batch orders (Karacal, 1997b). The customer orders are broken into detailed shop, work-center, and batch orders in a hierarchical fashion as they are released by decision making entities to subordinate entities.

\subsection{System level planning}

The instances of the systemPlanning knowledge base first checks the timing of the product order through a series of rules upon receiving a consultation request from a system control object. If timing is critically close to due-date, it 
immediately translates the product order into component/sub-assembly orders that are released to the relevant shop control entities. Otherwise, it evaluates a set of rules using order size, product type and customer identification to derive the importance level for the order. The next step in knowledge processing is the determination of the timing priority for the order using order importance, lead and slack time information. Then, the systemPlanning object acquires the present shop and capacity knowledge from the model in real time while the simulation is running. The following is the basic structure and some example rules of the Smalltalk based example systemPlanning knowledge base.

systemPlanning knowledge base

\section{Parameters:}

action, customer, order, productionLeadTime , product , quantity, rangeOne, rangeTwo shopCapacityStatus, shopLoadStatus, slack, SlackMinusLeadTime, timing

Rules: emergencyRelease

if: (slack $\leq$ slackMinusLeadTime) importantOrders then: [action is:'release' withCertainty: 1.0$]$

if: (slackMinusLeadTime $\leq$ rangeOne $\&($ order $=$ 'important')) then: [timing is: 'urgent' withCertainty: 0.95].

if: (slackMinusLeadTime $>$ rangeOne \& (slackMinusLeadTime $\leq$ rangeTwo) \&

$$
\text { (order ='Important')) }
$$

then: [timing is: 'normal' withCeratinty: 0.85].

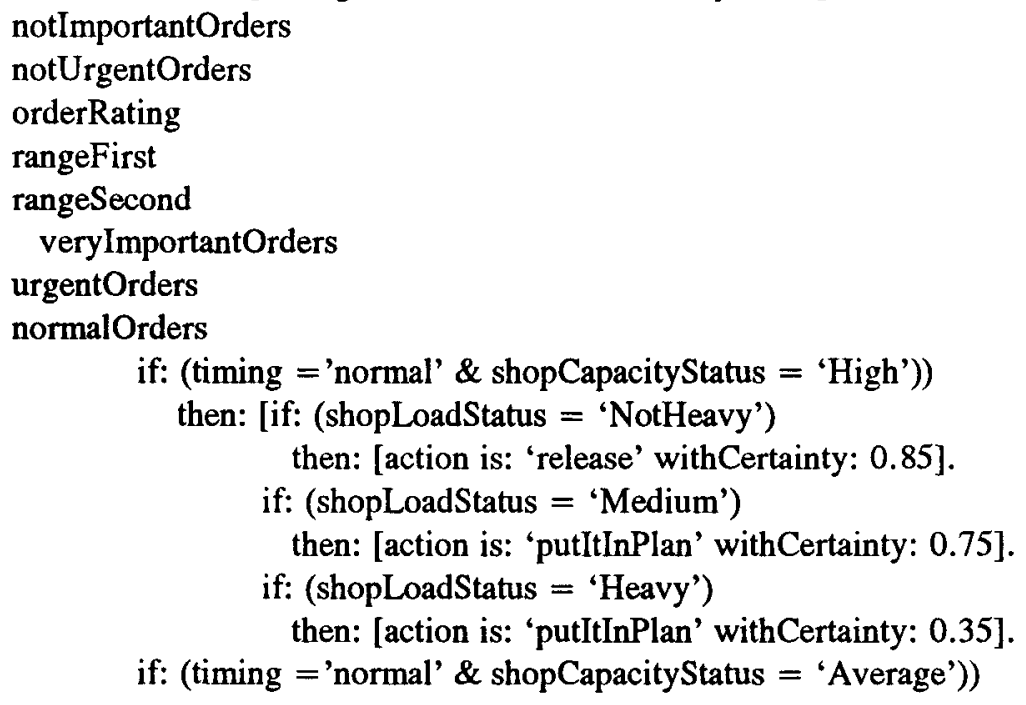




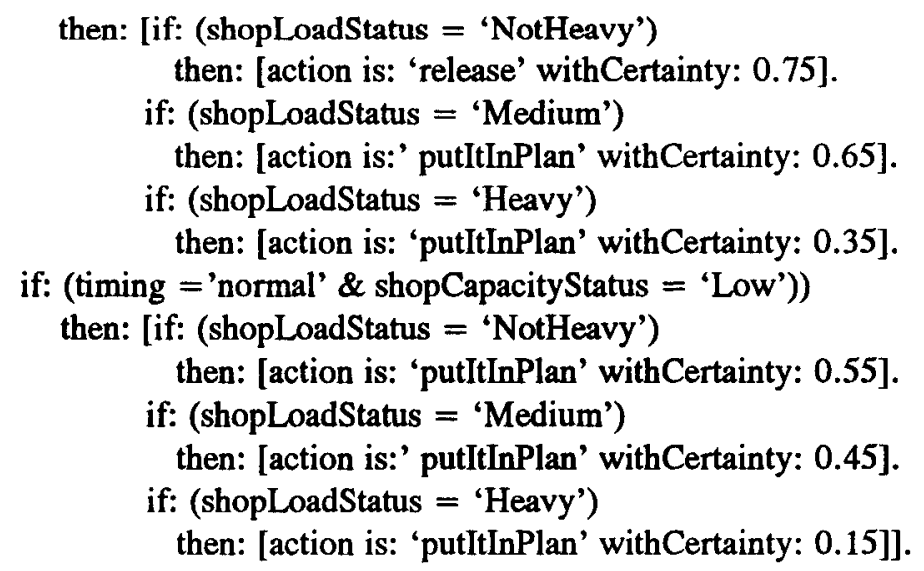

According to the timing of the order and the shop capacity at a given time, the normalOrders rule of the systemPlanning knowledge base given above evaluates all combinations with shop load status and finds an action and its certainty level. During this process, the quantitative information on shop loads and capacities are dynamically retrieved by the system control object from the shop control objects involved and converted into symbolic form in real simulation time. The conversion process takes place as follows:

1) The system controller looks at the shop load in terms of a moving monthly time bucket. As product orders are released, the bucket of the shop involved are loaded with orders using the lead time and quantity information.

As breakdowns/problems, changes in plan are encountered, the time buckets are updated in real time to reflect the changes. When a shop load status is requested for a particular shop, a quantitative expression that relates present shop load, unused time, and a flexibility factor is evaluated to find a numeric value at that instant of time. Depending on which predefined range this value falls into, one of the possible symbolic values such as high, low, etc. is returned to the knowledge base.

2) A symbolic value for the shop capacity status is determined using another expression that relates the number of operational machines, the type of orders being processed at that instant of time, shop reliability, and total number of available machines in the shop. Again, depending upon which range this value falls into, a symbolic value such as full-capacity, low-capacity, etc. is returned to the knowledge base.

Once the systemPlanning knowledge base finds a value to the parameter action, the value is passed back to the system controller object that implements the action by updating the internal and external states. The rules try to evenly spread the work load in terms of shop loads and order timing. The planning scheme uses a simple non-monotonic reasoning mechanism based on goal regression and is explained in detail in (Karacal, 1997b). 


\subsection{Shop level planning}

The next layer of knowledge bases, an instance of the shopPlanning knowledge base for each shop, are defined for non-programmed shop planning decisions. They are customized from their default structure by the model developer through definition of new parameters and rules. These knowledge bases deal with component orders and their main design principles are similar to those in systemPlanning. When they are interrogated, these knowledge bases asses a symbolic value for component order timing using due-date and lead time information from the interrogating shop control object. A set of rules classify the component on hand according to its importance and position in the product structure tree. The symbolic knowledge regarding the load and capacity status of the work-centers involved in processing of the present component order are obtained in real simulation time. The methods used in deriving these symbolic values are very similar to the ones used in the systemPlaning knowledge base, with the main difference being the weekly time bucket used. These knowledge bases search through a set of rules representing various combinations of work-center load and capacity status values, order timing, and the present shop plan to find a value for parameter action. This value is passed back to the shop controller which in turn implements the action.

\subsection{Work-center level planning}

The last non-programmed decision level in the hierarchy is handled by a set of workCenterPlaning knowledge bases that deal with batch orders released to machine controllers. These are relatively simple structures that decide on what to do with each individual batch order using the real time symbolic values derived for order timing, machine status, machine reliability, and present work-center plan. The work-center control object in turn implements the action returned by its workCenterPlaning knowledge base.

When physical machines receive batch orders from work-center control objects, they translate them into a set of activity orders that are carried out sequentially based on a programmed control that may perform condition checks such as physical material being available at that particular location, etc. This is where nonprogrammed control links to programmed control and decision processes are connected to physical activities.

The other model relevant data such as product order arrival patterns, customer types, order sizes, requested due-dates, processing times, MTTB, and MTTR are all specified by the model developer during model definition using the standard probability distributions provided in the system. 
The developed Smalltalk based simulation environment is tested through a simple make-to-order production system. The example with its system, shop, and workcenter planning entities is simulated for three months period (43,200 min. with 480 min./day). A one factor four levels experiment is designed to compare the performance of the system with or without different levels of knowledge based control. Considering the make-to-order nature of the manufacturing system, the following three statistics are analyzed.

1) Throughput time: Time between the release of a product order by the system controller and the completion of that order.

2) Order lateness: Time difference between the completion of an order and its due-date.

3) Customer response time: Time between the arrival of a customer to the system and the completion time for all the products requested by the customer in that order.

In addition, traditional simulation statistics such as machine utilizations and queue statistics are collected. Throughput time is selected as the main statistic of interest.

\subsection{Experimental levels}

The four experimental levels are defined as follow:

1) Manufacturing system without non-programmed control. The orders at all levels are translated into suborders and immediately released to the next level. The orders mostly accumulate at the machine control level where they are processed according to FIFO.

2) Only system level non-programmed control is employed using systemPlanning knowledge base. The lower level controls are programmed decisions and orders are processed using FIFO.

3) The system and shop control object both use their knowledge bases simultaneously.

4) The system, shop, and work-center control objects all use their knowledge bases simultaneously.

The main idea behind this experimental design is to perform a tighter timing and status control around product, component, and batch orders as they move downward in the control hierarchy. The frequency of consultations with the relevant knowledge bases during the simulation is different for each control level. The work-center knowledge bases are the most frequently queried ones, shop knowledge bases next, and the system knowledge base is the least. Five 
replications are made for each level. Common random number seeds are used to reduce variance during pair wise comparison of the difference between throughput times (in minutes) of levels. The random number seeds are changed between replications but kept the same across the levels. Furthermore, to better observe the effect of non-programmed control, the manufacturing system is slightly overloaded.

\subsection{Output analysis}

The simulation output is analyzed as follow: First, level 1 is compared with level 2 by taking the difference between mean throughput times of five replication pairs. Then, a $95 \%$ confidence interval is constructed for the true mean difference. The same procedure is applied for pair-wise comparison of other levels.

The pair-wise statistical analysis of the simulation output yielded the following conclusions.

Levels 1 - 2: The confidence interval for the true mean difference does not contain zero and completely lies in the positive region. Therefore, the difference is statistically significant and the system yields smaller mean throughput values when system level non-programmed control is employed.

Levels 2 - 3: The confidence interval for the true mean difference contains zero, implying that there is not enough statistical evidence to claim that one level is better than the other. The length of the interval suggests that more replications needed to reach a conclusion. Therefore, we can not claim that system performs better when shop and system level non-programmed control are employed simultaneously. Main reason for this conclusion is that the performance of the system heavily depends on the particular knowledge bases designed. Since this pair wise comparison did not yield a preference between the levels, levels 2 and 3 are pair-wise compared to level 4.

Levels 2 - 4: Although relatively wide, the confidence interval for the true mean difference does not contain zero and lies in the positive values region. Therefore, with the sample runs on hand we can claim that the throughput time is shorter when system, shop, and work-center levels use their knowledge bases simultaneously.

Levels 3 - 4: The confidence interval for the true mean throughput time difference lies in the right hand side of zero. Thus, the system yields shorter throughput times when all knowledge bases are employed simultaneously.

\section{CONCLUSIONS}

From the above statistical analysis, it is concluded that system performs best when non-programmed control is used at all control levels. This conclusion was 
somewhat expected and suggests that unless a sound non-programmed and programmed control scheme is applied at the shop floor level, the benefits that will be obtained from upper level non-programmed control will diminish and will not significantly impact the overall performance of the system. The other statistics collected such as customer response time and order lateness showed a similar trend. In general, throughput time can be improved by using shortest processing time (SPT) rule in programmed control, but since it does not take due-date information into account, it may severely deteriorate order lateness statistics. By using due-date, lead time, present state of the system together in the knowledge bases, we can define rules that make measured compromises among conflicting aspects of several system performance measures. Although not statistically analyzed, traditional measures such as utilization statistics and queue statistics showed that as layers of non-programmed hierarchical control are added, queue lengths were shorter and machine utilizations gave smaller values due to better batch timing.

The other possible set of experiments such as making replications with only shop or work-center knowledge base or other combinations to see combined and/or stand alone effects of the knowledge bases is left as a future investigation area. Also, other programmed control rules such as LIFO, STP, other control heuristics, and more refined knowledge bases and inference nules will be investigated in the future.

Another major advantage of this modeling framework developed is the flexibility it brings into the simulation and modeling process. The operations of both physical and logical aspects of a manufacturing system modeled not only closely represents reality but, are very modular and easy to change. This system can easily be used as a demonstration tool to show that various local optimization rules and procedures of different control levels (or even different modules of the same level), when put together, may deteriorate total system performance. It can also be a very useful tool to shown how conflicting multiple objectives of different control levels interact and affect global performance. The system will allow the design of operational policies and rules at each control level that will best fit overall system goals. The developed simulation tool is compared against traditional simulation approaches, where only physical entities with their implicit control structures are simulated, using Analytic Hierarchy Process (AHP) (Karacal et. al, 1996b). The results of the AHP study clearly indicate that this new approach is preferable to the traditional approach.

REFERENCES

Karacal, S. C., and J. Mize (1996a) A Formal Structure for Discrete Event Simulation, Part I: Modeling Multiple

Level Systems. IIE Transactions, 28, 753-760, 1996. 
Karacal, S. C., T. Beaumariage, Z. Karacal (1996b) Comparison of Simulation Environments Through Analytic

Hierarchy Process. Proceedings of 1996 Winter Simulation Conference, San Diego, CA

Karacal, S. C., and J. Mize (1997a) A Formal Structure for Discrete Event Simulation, Part II: Object Oriented

Software Implementation for Manufacturing Systems. will appear in IIE Transactions, 1997.

Karacal, S. C. (1997b) A goal Decomposition Language for Manufacturing System Simulation. Proceedings of IE

Research Conference, 1997, Miami Beach, FL.

Karacal, S. C. (1997c) A Formal Structure for Discrete Event Simulation, Part III: Knowledge Processing During

Simulation. Working technical paper, 97-2, SIUE, Industrial Engineering, Edwardsville, $\mathrm{IL}$, working technical

paper 97-2.

\section{BIOGRAPHY}

S. Cem Karacal is an assistant professor of Industrial Engineering at Southern Illinois University at Edwardsville. He received his B.S. degree in IE from Orta Dogu Teknik Universitesi, Turkey, in 1982, and M.S. and Ph.D. degrees in IE from Oklahoma State University in 1986 and 1991, respectively. He has tree years of experience in industry and consulting. His primary areas of interest are object oriented modeling methodologies, AI applications in simulation, and semiconductor manufacturing scheduling. He is a member of IIE, SME, Alpha Pi $\mathrm{Mu}$, and Tau Beta Pi. 